\title{
FISIOLOGIA ALIMENTAR DO BERBIGÃO ANOMALOCARDIA BRASILIANA (GMELIN, 1791) (MOLLUSCA: BIVALVIA)
}

\author{
CHARRID RESGALLA JR ${ }^{1,2} \&$ ANDRESSA DE CAMARGO PIOVEZAN ${ }^{1}$ \\ ${ }^{1}$ CTTMar - Universidade do Vale do Itajaí, UNIVALI. Caixa Ppstal 360, Itajaí, SC - CEP 88.302-202 - Brasil .²resgalla@univali.br
}

\section{RESUMO}

A fisiologia alimentar de bivalves filtradores tem sido pouco investigada para as espécies nativas do Brasil. Entretanto, estas informações são fundamentais nos futuros estudos de cultivo e interpretação das características ecológicas das espécies. Entre as espécies de moluscos infaunais, o bivalve Anomalocardia brasiliana, conhecido popularmente como berbigão, apresenta grande interesse comercial e pouco é conhecido sobre as suas taxas alimentares. Este trabalho apresenta os resultados de quatro experimentos realizados em laboratório utilizando diferentes classes de tamanho de $A$. brasiliana coletadas em diferentes épocas do ano. A partir dos dados de séston natural, massa de fezes e pseudofezes produzidas e seus respectivos conteúdos orgânicos e inorgânicos foram estimados as taxas de clareamento, filtração, rejeição, ingestão e a eficiência de absorção pelo método de biodepósitos. Observou-se que o berbigão $A$. brasiliana apresenta comportamento similar à grande maioria de bivalves infaunais investigados no mundo. Entretanto, sua taxa de rejeição mostrou-se independente com a variação do séston o que leva a uma baixa taxa de ingestão em concentrações de séston inferiores a 10 $\mathrm{mg} \cdot \mathrm{L}^{-1}$. A baixa capacidade de selecionar a matéria orgânica presente no séston foi atribuído ao alto conteúdo orgânico presente no material particulado em suspensão da Baía Sul da llha de Santa Catarina.

PALAVRAS CHAVE: Anomalocardia brasiliana, Berbigão, Taxas alimentares, Biodepósitos.

\section{ABSTRACT}

Feeding physiology of the Anomalocardia brasiliana (Gmelin, 1791) (Mollusca: Bivalvia)

Little investigation has been carried out on the feeding physiology of filter-feeding bivalves in native species of Brazil. However, this information is essential for future studies on its cultivation, and for the interpretation of ecological characteristics of the species. Among the infaunal mollusk species, the bivalve Anomalocardia brasiliana is of great commercial interest, though little is known about its feeding rate. This work presents the results of four experiments carried out using different size classes of $A$. brasiliana collected at different times of the year. Based on the data for natural seston, mass of feces and pseudofeces produced and its respective organic and inorganic contents, the clearance rates, filtration, rejection, ingestion and absorption efficiency were estimated by the biodeposit method. It was observed that the $A$. brasiliana presents similar behavior to the majority of infaunal bivalves investigated worldwide. However, its rejection rate proved to be independent of seston variation, which leads to a low ingestion rate at seston concentrations below $10 \mathrm{mg} \cdot \mathrm{L}^{-1}$. The low capacity to select the organic matter present in the seston was attributed to the high organic content present in the particulate matter in suspension in the Baía Sul of the llha de Santa Catarina.

KEY WORDS: Anomalocardia brasiliana, Feeding rates, Biodeposits, Santa Catarina.

\section{INTRODUÇÃO}

O molusco Anomalocardia brasiliana, conhecido popularmente como "berbigão", "papafumo", "sarnambi" ou "vôngoli", pertence à família Veneridae com várias espécies de interesse comercial em todo o mundo. Este organismo é explorado artesanalmente em diversas regiões do país, para consumo de subsistência e venda ao mercado consumidor. São preferencialmente encontrados em fundos arenosos/lodosos (Rios 1994), localizados em enseadas, baías e estuários, com uma distribuição entre as Antilhas e o Uruguai.

É um bivalve eurihalino, euritérmico (SchaefferNovelli 1976) e com alta taxa de crescimento (Pezzuto \& Echternacht 1999). Diferentes estudos já foram realizados com a espécie destacando-se os trabalhos de distribuição, abundância e dinâmica populacional (Shaeffer-Novelli 1976 e 1980, Peso 1980; Pezzuto \& Echternacht 1999, Boehs 2000); reprodução (Narchi 1976, Grotta \& Lunetta 1980 e
1982, Boehs 2000, Araujo 2001); desenvolvimento embrionário (Monti et al. 1999) e morfologia funcional (Narchi 1972).

Em Santa Catarina, o Saco dos Limões, localizado na margem leste da Baía Sul da llha de Santa Catarina (município de Florianópolis), é onde a captura da espécie desenvolveu-se de forma intensa ao longo dos anos, caracterizando a área como sendo uma das mais importantes quanto à extração do berbigão no Brasil. Este fato levou o inicio de estudos sobre a biologia e pesca do berbigão pelo IBAMA a partir de 1988 (Pezzuto \& Echternacht, 1999). Foi a partir destes estudos, e por sua relevância, que foi criada a primeira Reserva Extrativista Marinha brasileira, denominada Reserva Extrativista Marinha do Pirajubaé (REMAPI) com cerca de 240 hectares referentes a uma zona de baixio (Fig. 1).

Segundo Silva e Souza (2003) a partir de 2001 foi realizado um mapeamento do recurso em 
toda área do baixio onde foi observado que as maiores biomassas do berbigão encontram-se na área mais externa, o que provavelmente afeta de maneira negativa os organismos da área mais interna por exaustão do séston devido à atividade de clareamento e filtração. Para entender este processo, seria necessário o conhecimento das taxas fisiológicas alimentares desta espécie além de informações sobre a variabilidade do séston no ambiente.

A influência da quantidade e qualidade do alimento sobre as taxas fisiológicas de bivalves é, sem dúvida, o fator ambiental mais importante e o mais exaustivamente investigado por diferentes grupos de pesquisa no mundo (Griffiths \& Griffiths 1987). A quantidade de alimento disponível no ambiente e o seu conteúdo calórico (orgânico) são os forçantes primários para as adaptações fisiológicas dos organismos na conquista de diferentes ambientes assim como na determinação de suas taxas de crescimento (Bayne \& Newell 1983).

Por outro lado, as respostas das taxas fisiológicas frente às variações do alimento apresentam igualmente uma ampla variabilidade de resultados, devido, principalmente, às condições de execução dos experimentos em laboratório. A partir da década de 70 , até os dias de hoje, variações na metodologia de estudo das taxas fisiológicas de bivalves envolveram desde o comportamento destes organismos alimentados com culturas mono específicas de microalgas, passando por misturas com silte e sedimentos, chegando aos estudos com o séston natural realizados no próprio ambiente. O objetivo desta evolução seria a procura do comportamento fisiológico e de balanço energético o mais próximo da realidade (Iglesias et al. 1998).

A partir de 1996, com os trabalhos de Hawkins et al. (1996) e Iglesias et al. (1996), entre outros, utilizando técnicas de biodepósitos (produção de fezes e pseudofezes), as estimativas das taxas de clareamento e da eficiência de absorção ficaram mais realistas e permitiram um maior detalhamento de subunidades nos processos de alimentação e de obtenção de energia para os organismos.

Com esta nova técnica de estudo da fisiologia alimentar, vários trabalhos têm apontado diferenças significativas entre bivalves infaunais com os epifaunais (Hawkins et al. 1980, Prins \& Smaal 1989, Prins et al. 1991, Smaal et al. 1997). No Brasil estudos das taxas alimentares do mexilhão Perna perna (espécie epifaunal) já têm sido executados por Mello (1999); Schmitt (2002) e Suplicy et al. (2003) utilizando a técnica de biodepósitos. Para espécies infaunais nada ainda tem sido executado até o momento.

Este trabalho apresenta informações a respeito da fisiologia alimentar de $A$. brasiliana, afim de que, somadas às demais informações de sua biologia e ecologia, podem fornecer subsídios para um conhecimento da espécie, seu cultivo e manejo de estoques naturais.

\section{MATERIAL E MÉTODOS}

\section{Coleta dos organismos}

Foram coletados 10 exemplares do berbigão Anomalocardia brasiliana, em 3 diferentes classes de tamanho ( $<20 \mathrm{~mm}, 20-25 \mathrm{~mm}$ e $>25 \mathrm{~mm}$ de tamanho padrão), obtidos na Reserva Extrativista do Pirajubaé (REMAPI; Florianópolis/SC, LAT 27ㅇ 38' $00^{\prime \prime} \mathrm{S}$, LONG $48^{\circ} 33^{\prime} 05^{\prime \prime} \mathrm{W}$ ) (Fig. 1). As coletas foram realizadas nos meses de maio/julho/agosto de $2004 \mathrm{e}$ agosto de 2005. Na mesma ocasião foram coletados 160 litros de água do ambiente para os experimentos laboratoriais, utilizando o séston natural (material particulado em suspensão). Os dados de temperatura foram obtidos no momento da coleta com o uso de um termômetro de coluna de mercúrio e, os dados de salinidade foram obtidos em laboratório, com o uso de um condutivímetro Orion. 


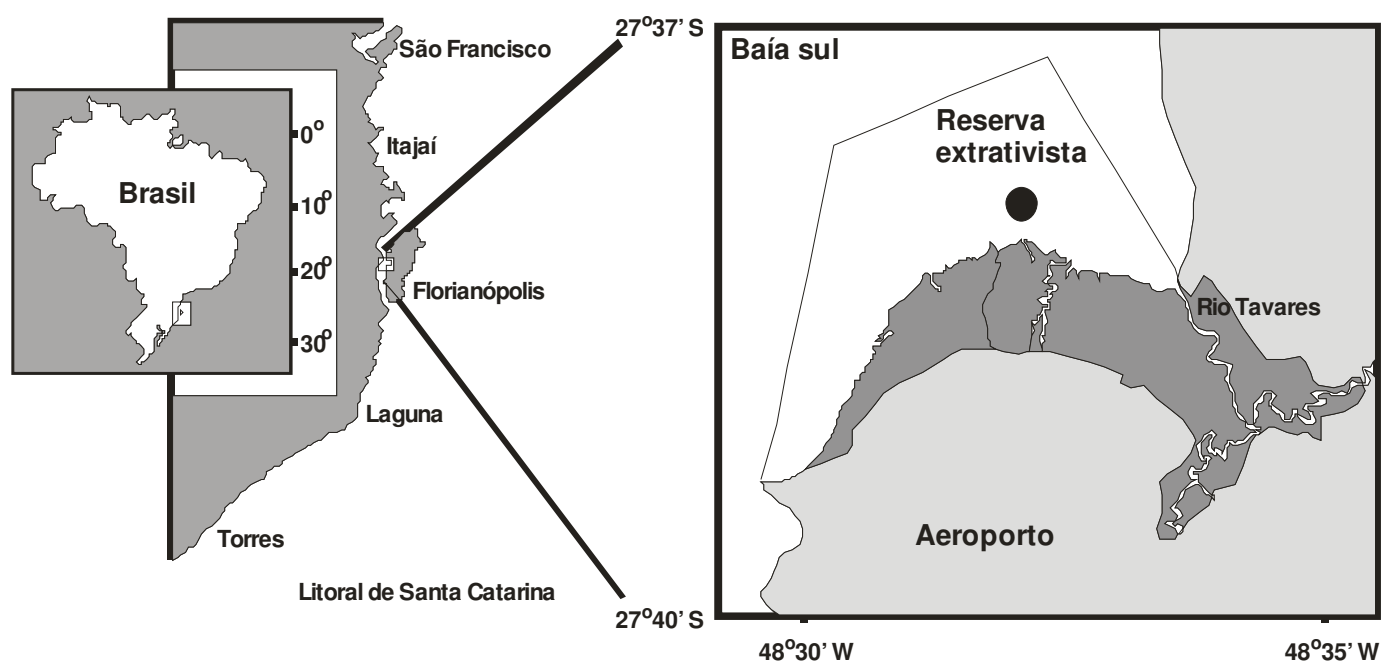

Figura 1 - Mapa da área da Reserva Extrativista do Pirajubaé, Ilha de Santa Catarina (Florianópolis, SC), e ponto $(\bullet)$ de extração do berbigão Anomalocardia brasiliana.

\section{Preparo dos organismos}

O transporte dos organismos até o Laboratório de Ecotoxicologia do CTTMar/UNIVALI foi realizado em caixa térmica e fina lâmina de água para manter a umidade. Ao chegarem no laboratório, os berbigões foram mantidos por $12 \mathrm{~h}$ em água do mar filtrada em filtro de $0,5 \mathrm{~mm}$, em fotoperíodo de $12 \mathrm{~h}$ claro e $12 \mathrm{~h}$ escuro à temperatura constante de $20 \pm 2^{\circ} \mathrm{C}$ e sem sedimento, para a limpeza do trato digestivo antes dos experimentos.

\section{Condição experimental}

Após o período de manutenção, os organismos foram acondicionados individualmente em placa de Petri e estas em bandejas plástica de 45 litros alimentada com a água do próprio ambiente e séston natural em sistema de fluxo contínuo na taxa de 15 L.h $h^{-1}$. Os organismos foram mantidos nestas condições para a produção de fezes e pseudofezes por 5 a 6 horas (em função da quantidade de material produzido). Os experimentos foram executados a 20 $\pm 2^{\circ} \mathrm{C}$, luz constante e sem sedimento.

Os organismos que não abriram suas valvas ou não produziram fezes ou pseudofezes no período de aclimatação (1 hora) foram descartados do experimento. Devido a este comportamento, o experimento 1 apresentou um número amostral (n) de 11 organismos, experimento 2 com $n=6$, experimento 3 com $n=3$ (retirado das análises devido ao baixo número) e experimento $4 \mathrm{com} n=18$.

\section{Parâmetros}

A cada hora experimental, as fezes e pseudofezes foram coletadas separadamente com conta gotas em plástico de ponta fina e de $3 \mathrm{~mL}$ e acondicionadas em tubo de ensaio para a determinação de seu peso seco e conteúdo orgânico e inorgânico. Para o séston natural, foi utilizado, no mínimo, 1 litro de amostra da água do ambiente.

O procedimento padrão para estimar o peso seco, peso em matéria orgânica e inorgânica das fezes, pseudofezes e séston foi a filtragem em filtros GF/F pré-calcinados e tarados; lavagem com água destilada para a remoção de sais, secagem dos filtros em estufa a $60^{\circ} \mathrm{C}$ por 24 horas para a determinação de seu peso seco e posterior queima dos filtros em forno mufla a $450-500{ }^{\circ} \mathrm{C}$ por $2 \mathrm{~h}$ para a determinação do peso seco livre de cinzas ou peso em matéria orgânica.

\section{Taxas fisiológicas}

As taxas fisiológicas alimentares do berbigão $A$. brasiliana foram estimadas segundo a metodologia proposta por Navarro et al. (1991) e Iglesias et al. (1996) em base de coleta dos biodepósitos (fezes e pseudofezes) além do uso do material particulado total da água natural do ambiente. A nomenclatura e parâmetros utilizados para as estimativas das taxas apresentam a terminologia original na língua inglesa. 


\section{$\underline{\text { Taxa de clareamento }}$}

$$
C R=\frac{\text { PIM }_{B}}{\text { PIM }_{W}}
$$

Sendo:

$\mathrm{CR}=$ Taxa de clareamento $\left(\right.$ L. $\left.\mathrm{h}^{-1}\right)$

$\mathrm{PIM}_{\mathrm{B}}=$ matéria particulada inorgânica dos biodepósitos (fezes + pseudofezes) $\left(\mathrm{mg} \cdot \mathrm{h}^{-1}\right.$ )

$\mathrm{PIM}_{\mathrm{w}}=$ matéria particulada inorgânica na água $\left(\mathrm{mg} \cdot \mathrm{L}^{-1}\right)$

\section{Taxa de filtracão:}

$F R=T P M_{W} \times C R$

Sendo:

$\mathrm{FR}=$ Taxa de filtração $\left(\mathrm{mg} \cdot \mathrm{h}^{-1}\right)$

$\mathrm{TPM}_{\mathrm{w}}=$ Material particulado total na água $\left(\mathrm{mg} \cdot \mathrm{L}^{-1}\right)$

$\mathrm{CR}=$ Taxa de clareamento $\left(\right.$ L. $\left.\mathrm{h}^{-1}\right)$

\section{Taxa de ingestão:}

$I R=F R-R R$

Sendo:

$\mathrm{IR}=$ Taxa de ingestão $\left(\mathrm{mg} \cdot \mathrm{h}^{-1}\right)$

$\mathrm{FR}=$ Taxa de filtração $\left(\mathrm{mg} \cdot \mathrm{h}^{-1}\right)$

$\mathrm{RR}=$ Taxa de rejeição (pseudofezes) $\left(\mathrm{mg}^{\mathrm{h}} \mathrm{h}^{-1}\right)$

\section{Taxa de egestão}

$E R=I E R+O E R$

Sendo:

$E R=$ Taxa de egestão $\left(m g \cdot h^{-1}\right)$

IER= Taxa de egestão inorgânica (fezes) $\left(\mathrm{mg}^{\mathrm{h}} \mathrm{h}^{-1}\right)$

OER = Taxa de egestão orgânica (fezes) $\left(\mathrm{mg}^{\mathrm{h}} \mathrm{h}^{-1}\right)$

\section{Eficiência de absorcão orgânica}

$O A E=\frac{(f-p)}{(1-p) f}$

Sendo:

OAE = Eficiência de absorção orgânica (\%)

$f=$ Porcentagem orgânica do $\operatorname{TPM}_{\mathrm{W}}(\%)$

$p=$ Porcentagem orgânica das fezes (\%)

\section{Padronização das taxas fisiológicas}

O peso seco dos organismos utilizados nos experimentos foi estimado mediante a dissecação dos tecidos das conchas e secagem das partes moles a $60{ }^{\circ} \mathrm{C}$ por 48 horas. Foi realizado a padronização das taxas fisiológicas para um peso seco médio padrão de $0,14 \mathrm{~g}$ (média dos organismos utilizados nos experimentos com um desvio padrão de 0,035 g). Para a padronização das taxas foi utilizado o índice $\boldsymbol{b}$ da equação alométrica $\left(Y=a X^{b}\right)$, obtido através da regressão linear da taxa de clareamento $(Y)$ com o peso seco do organismo $(X)$. A equação utilizada para a padronização das taxas seguiu a proposta por Bayne et al. (1985):

$\log Y c=\log Y_{0}-\left(b \log X_{0}-b \log X c\right)$

Sendo:

Yc é a taxa fisiológica padronizada pelo peso padrão $(0,14 \mathrm{~g})$;

Yo é a taxa medida;

Xo o peso do organismo, e

Xc o peso padrão $(0,14 \mathrm{~g})$.

\section{Análises estatísticas}

As regressões entre 0 peso seco dos organismos $e$ as taxas de clareamento foram validadas por análise de regressão para um nível de significância de $p<0,05$ após a transformação dos dados em LOG para a linearização da curva. Para as comparações múltiplas entre as taxas de clareamento $(C R)$, filtração (FR), rejeição (RR), egestão (ER) e ingestão (IR) com a variação do material particulado em suspensão (séston, TPMw) e entre a eficiência de absorção orgânica (OAE) com a fração orgânica no séston $(f)$, foram realizadas análises de variância (ANOVA) após a transformação dos dados em LOG para a sua normalização e homogeneidade utilizando o programa TOXSTAT (versão 3.3).

\section{RESULTADOS}

\section{Parâmetros ambientais}

$\mathrm{Na}$ Tabela 1 são apresentadas as datas de coleta dos quatro experimentos realizados com o berbigão, assim como dados de salinidade, temperatura, séston e porcentagem de matéria orgânica do séston no momento da coleta dos organismos. Observou-se que a salinidade ficou praticamente constante enquanto que a temperatura mostrou uma maior amplitude de variação entre os períodos amostrais. Destaca-se, entretanto, uma relação inversa entre a concentração do séston com o seu conteúdo em matéria orgânica. 
Tabela 1 - Datas dos experimentos e valores de salinidade, temperatura, séston (TPMW) e porcentagem orgânica do séston (f) para a água nos períodos de coleta de berbigão utilizados nos experimentos laboratoriais.

\begin{tabular}{cccccc}
\hline Experimento & Data & Salinidade & Temperatura & TPMw $^{\left(m g . L^{-1}\right)}$ & $f(\%)$ \\
\hline 1 & $03 / 05 / 2004$ & 31,3 & 22,2 & 10,95 & 23,1 \\
2 & $12 / 07 / 2004$ & 30,1 & 16,5 & 3,20 & 31,4 \\
3 & $31 / 08 / 2004$ & 29,8 & 18,2 & 7,70 & 24,4 \\
4 & $27 / 08 / 2005$ & 30,2 & 19,3 & 21,89 & 18,9 \\
\hline
\end{tabular}

Neste estudo, o séston (TPMw) variou de 3,2 a $21,9 \mathrm{mg} \cdot \mathrm{L}^{-1}$, enquanto o material particulado orgânico (POMw) oscilou de 1 a 4,13 mg..-1 , o material particulado inorgânico (PIMw) contido no séston foi de
2,2 a 17,75 mg. $\mathrm{L}^{-1}$ e a fração orgânica do séston $(f)$, relação POMw/TPMw, apresentou variação de 0,19 a 0,31 (Fig. 2).
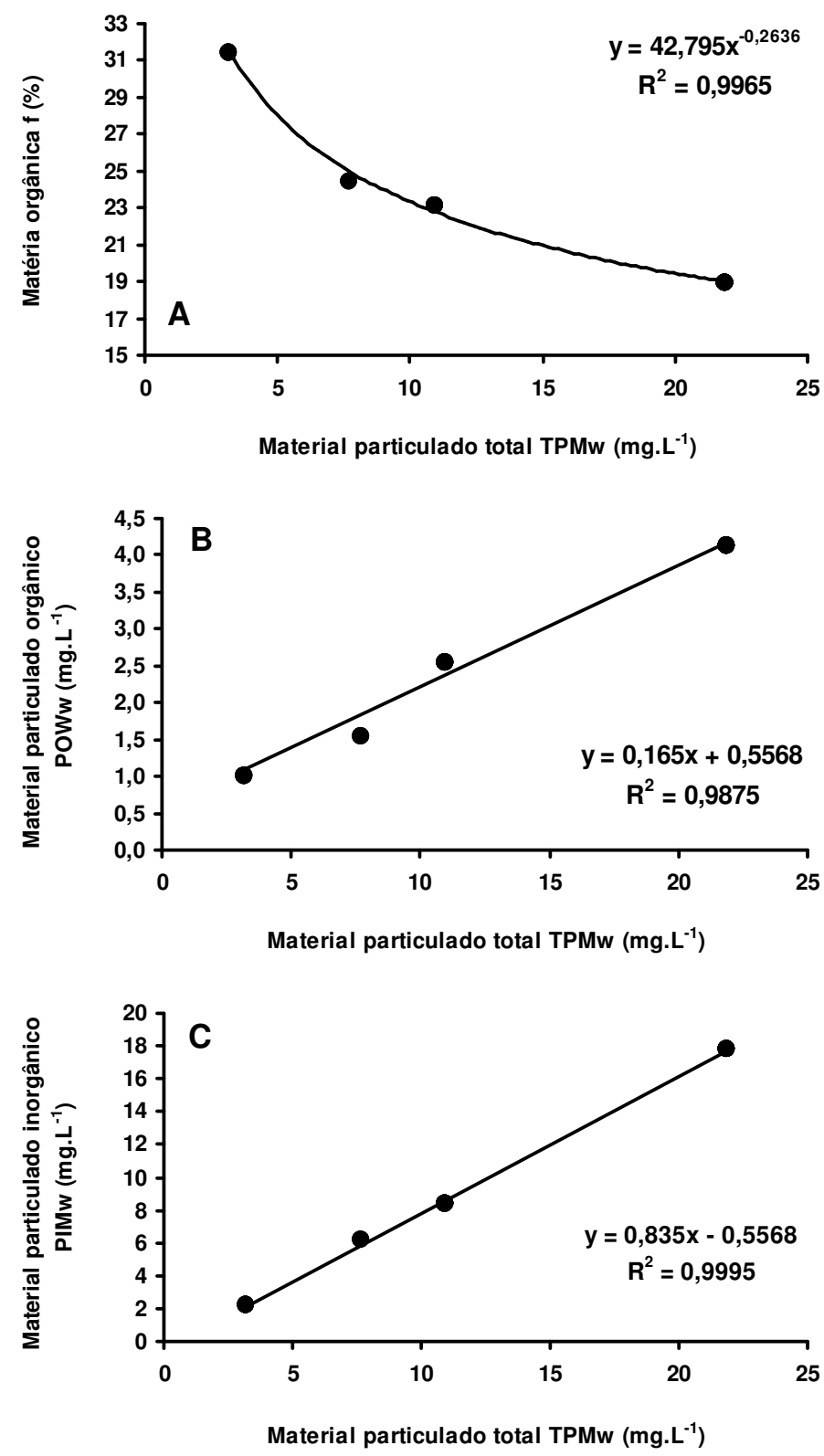

Figura 2 - A: Relação entre o séston (TPMw) e o seu conteúdo orgânico (f); B: Relação entre o séston (TPMw) e o material orgânico particulado (POWw) e C: Relação entre o séston (TPMw) e o material inorgânico particulado (PIMw). 


\section{Relação peso e taxa de clareamento}

Devido a problemas relacionados nas comparações entre as taxas fisiológicas e o peso dos organismos é freqüente o uso de uma padronização dos cálculos para um peso padrão eliminando variações intrínsecas entre organismos de mesmo tamanho, mas de peso diferentes. Para esta correção é utilizado o expoente $\boldsymbol{b}$ da equação alométrica que explica o quanto varia uma taxa em função do peso do organismo (Bayne et al. 1985). O índice adotado de 0,8 é resultado da média de dois experimentos que apresentaram correlação significativa (Tabela 2).

Tabela 2 - Valor de b da equação alométrica $\left(Y=a X^{b}\right)$, coeficiente de terminação e nível de significância para $\mathrm{p}<0.05$ da relação entre a taxa de clareamento $(\mathrm{Y}) \times$ peso seco do berbigão $(X)$.

\begin{tabular}{lccccc}
\hline Experimento & Número de organismos & $\mathbf{a}$ & $\mathbf{b}$ & $\mathbf{R}^{\mathbf{2}}$ & Significância \\
\hline 2 & 5 & 0,35 & 0,57 & 0,60 & 0,1226 \\
1 & 11 & 0,69 & 0,91 & 0,59 & $0,0056^{*}$ \\
4 & 18 & 0,25 & 0,69 & 0,54 & $0,0005^{*}$ \\
Média & & & 0,80 & & \\
\hline
\end{tabular}

*significância para $p<0,05$.

\section{Avaliação dos biodepósitos}

Foram analisados e comparados, os conteúdos orgânicos das fezes e pseudofezes em relação ao material particulado total (TPMw) e seu conteúdo orgânico (Tabela 3). Observou-se que os conteúdos orgânicos das pseudofezes apresentaram alto conteúdo orgânico, sendo inclusive maiores que o próprio TPMw. Este fato sugere que $A$. brasiliana apresenta uma baixa eficiência de seleção no processo de rejeição. Desta forma, a rejeição poderia ser utilizada pelo berbigão mais no controle quantitativo do alimento ingerido do que propriamente selecionar a fração orgânica e otimizar a sua eficiência de absorção.

Tabela 3 - Valores médios do séston (TPMw), seu conteúdo orgânico (f) e dos conteúdos orgânicos dos biodepósitos (fezes e pseudofezes).

\begin{tabular}{lcccc}
\hline Experimento & $\begin{array}{c}\text { Séston } \\
\text { (TPMw, mg. } \text { - }^{-1} \text { ) }\end{array}$ & $\begin{array}{c}f \text { (conteúdo orgânico do séston, } \\
\% \text { ) }\end{array}$ & $\begin{array}{c}\text { Conteúdo orgânico das } \\
\text { fezes }(\%)\end{array}$ & $\begin{array}{c}\text { Conteúdo orgânico das } \\
\text { pseudofezes }(\%)\end{array}$ \\
\hline 2 & 3,20 & 31,4 & 19,2 & 67,7 \\
1 & 10,95 & 23,1 & 7,4 & 43,2 \\
4 & 21,89 & 18,9 & 11,8 & 24,6 \\
\hline
\end{tabular}

\section{Comportamento das taxas alimentares em relação ao séston}

A taxa de clareamento apresentou uma significativa diminuição com o aumento do TPMw, e as taxas de filtração, ingestão e egestão aumentaram

significativamente com o TPMw (Fig. 3 e Tabela 4). Entretanto, a taxa de rejeição manteve-se constante e independente da concentração do séston, assim como a eficiência de absorção orgânica foi constante com o conteúdo orgânico do séston ( $f$ ).

Tabela 4 - Resultado da análise de variância (ANOVA) para comparação múltipla entre as taxas de clareamento $(C R)$, filtração $(F R)$, rejeição $(R R)$, ingestão (IR) e egestão (ER) em diferentes concentrações do séston (TPMw) e da eficiência de absorção orgânica (OAE) em diferentes percentuais de matéria orgânica do séston (TPMw) para A. brasiliana, com um nível de significância de $5 \%(\alpha=0,05)$. Onde: g.l. é o grau de liberdade; $F$ valor de Fisher e * sinaliza parâmetros com diferenças significativas $(p<0,05)$.

\begin{tabular}{|l|c|c|c|c|}
\hline Variáveis & g.l. & F crítico & F calculado & Significância \\
\hline CR $\times$ TPMw & $(2,30)$ & 3,32 & 11,53 & ${ }^{*}$ \\
\hline FR $\times$ TPMw & $(2,30)$ & 3,32 & 20,75 & ${ }^{*}$ \\
\hline RR $\times$ TPMw & $(2,30)$ & 3,32 & 1,54 & \\
\hline IR $\times$ TPMw & $(2,30)$ & 3,32 & 31,41 & ${ }^{*}$ \\
\hline ER $\times$ TPMw & $(2,30)$ & 3,32 & 14,18 & ${ }^{*}$ \\
\hline OAE $\times f$ & $(2,25)$ & 3,39 & 0,51 & \\
\hline
\end{tabular}



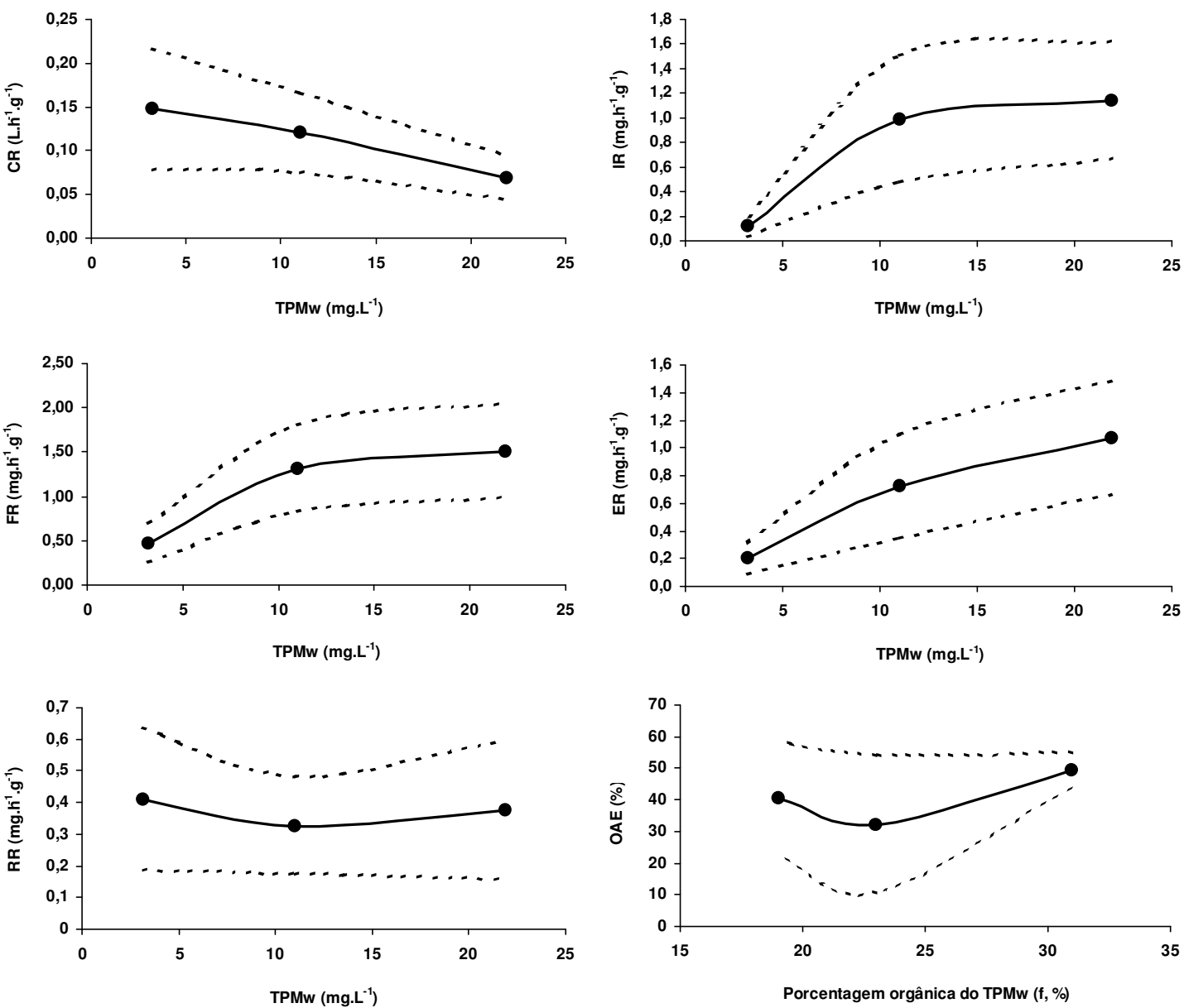

Figura 3 - Linhas de tendência da média (contínuo com ponto) e do desvio padrão (linha tracejada) das taxas de clareamento (CR), filtração (FR); rejeição (RR); ingestão (IR), egestão (ER) em relação ao séston (TPMw) e da eficiência de absorção orgânica (OAE) em relação ao percentual orgânico do séston.

\section{DISCUSSÃO}

\section{Relação peso e taxa de clareamento}

Os trabalhos sobre a revisão de estudos das taxas fisiológicas de bivalves suspensívoros destacam que o valor de $\boldsymbol{b}$ varia de 0,4 a 0,8 para a relação entre peso e a taxa de clareamento (Bayne \& Newell 1983, Griffiths \& Griffiths 1987). Resgalla Jr. et al. (2006) obteve um valor de $\boldsymbol{b}$ de 0,48 para o mexilhão Perna perna. Para representantes da infauna, Bacon et al. (1998) utilizaram um valor de $\boldsymbol{b}$ de 0,68 para as taxas de clareamento de Mya arenaria enquanto que Iglesias et al. (1996) assumiram um valor de 0,57 para Cerastoderma edule. $O$ valor de 0,8 estimado para $A$. brasiliana neste trabalho é coerente com os observados na bibliografia.

\section{Taxas fisiológicas alimentares e o séston}

O material particulado em suspensão, fonte alimentar dos organismos filtradores, pode sofrer amplas variações em quantidade e qualidade na água do mar. Experimentos que avaliem essas variações são importantes para compreendermos as relações dos organismos suspensívoros com o fluxo de material particulado em ambientes costeiros. Muitas informações podem ser obtidas a partir de alterações na quantidade e proporção entre 0 material particulado (séston) e a matéria orgânica particulada disponível para os bivalves (Navarro et al. 1991, Iglesias et al. 1998).

Normalmente considera-se que as variações de temperatura e salinidade apresentam uma maior influência sobre as taxas alimentares de bivalvos, quando praticamente não existem variações no séston do ambiente, tanto em baixas como em altas 
concentrações (Resgalla Jr. et al. 2007, Schmitt, 2002). Como fonte de variação, considera-se a composição nutricional do séston, não somente a fração orgânica, mas também a concentração de pigmentos (clorofila e feopigmento), níveis de carbono e nitrogênio particulado e a bioquímica (carboidratos, lipídeos e proteínas). A origem da matéria orgânica pode ainda ser classificada como detrítica (matéria decomposta e bactérias) e/ou de fitoplâncton ou microfitobentos, alterando assim seu valor nutricional e influenciando a digestibilidade e teor energético (Navarro et al. 1991). São, portanto, os elementos integrados quali-quantitativos do séston (TPMw, PIMw, POMw e $f$ ) os fatores que de fato influenciam as taxas de alimentação e biodeposição.

Durante o processo de filtração, parte das partículas filtradas passa pelo processo de ingestão e é eliminada na forma compactada como fezes (processo de egestão), outra porção é rejeitada, aglomerada em muco, na forma de pseudofezes. Segundo Bayne et al. (1988), a formação de pseudofezes representa uma maneira de selecionar o material filtrado, mantendo a qualidade do material ingerido, mesmo com a variação da fração orgânica no séston. As brânquias e os palpos labiais separam a fração orgânica e inorgânica do séston, ingerindo a primeira e rejeitando na forma de pseudofezes a segunda.

As taxas de clareamento, filtração, ingestão e egestão de $A$. brasiliana apresentaram um comportamento padrão estabelecido para os bivalves filtradores quando comparada com a concentração do séston (TPMw) (Navarro \& Iglesias 1993, Iglesias et al. 1996, Bacon et al. 1998, MacDonald et al. 1998).

Entretanto, os resultados sugerem que $A$. brasiliana apresenta um comportamento diferenciado para as suas taxas de rejeição, mantendo-se constante e independente da concentração do séston. Para a grande maioria dos bivalves filtradores a taxa de rejeição apresenta comportamento similar a taxa de filtração, porem em menores valores (Navarro \& Iglesias 1993). Bacon et al. (1998), entretanto, cita para Mya arenaria comportamento similar para a taxa de rejeição apresentada neste trabalho. Devido às condições de execução apresentadas neste trabalho (em laboratório), recomenda-se que este comportamento seja ainda confirmado a partir de experimentação em campo.

Este comportamento diferenciado de $A$. brasiliana com relação a taxa de rejeição constante e independente da concentração do séston, alto conteúdo de matéria orgânica nas pseudofezes indicando pouca capacidade de seleção de partículas, pode estar relacionada as características do próprio séston do local de sua ocorrência. Segundo os modelos entre o séston e o seu conteúdo orgânico obtidos por Resgalla Jr. et al. (2007) para enseada da Armação do Itapocoroi (litoral norte de Santa Catarina), Suplicy et al. (2003) para a enseada do Brito e de Bombinhas (litoral centro de Santa Catarina) e os dados deste trabalho (Figura 2A), observou-se que o séston da região do baixio de ocorrência do berbigão do Saco dos Limões apresenta alto conteúdo de matéria orgânica provavelmente pela própria ressuspensão do fundo. Esta característica do séston poderia proporcionar ao berbigão uma baixa eficiência em termos alimentares, fugindo ao conceito de que a infauna sempre apresentaria uma maior capacidade na seleção de partículas em relação à epifauna (Bacon et al. 1998).

Outro parâmetro de comportamento diferenciado a grande maioria dos bivalvos filtradores refere-se a eficiência de absorção orgânica. Esta taxa normalmente apresenta forte dependência com o conteúdo orgânico do séston e é maior quanto maior for este conteúdo (Bayne \& Newell 1983, Griffiths \& Griffiths 1987). A eficiência obtida para $A$. brasiliana, similar ao observado para a taxa de rejeição, mostrou-se independente com a qualidade do séston. Entretanto, este resultado poderia ser diferente se a variabilidade entre as réplicas não fosse tão alta (Fig. 4). 


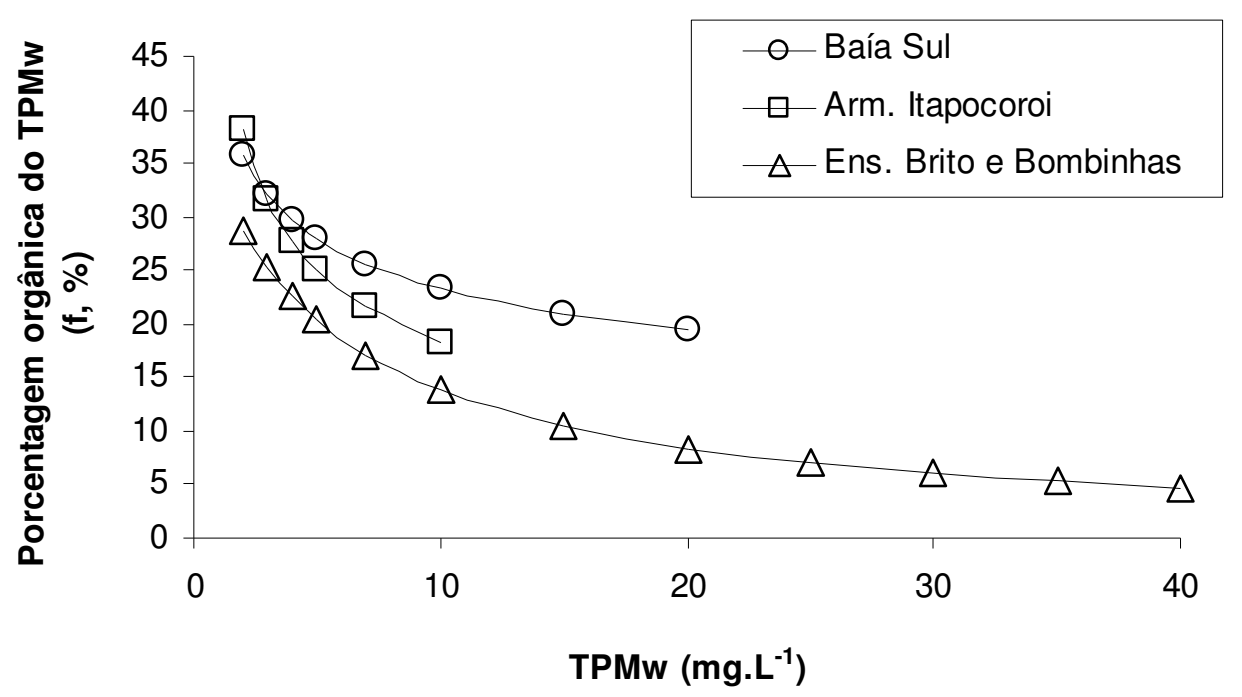

Figura 4 - Relação entre o séston (TPMw) e o seu conteúdo em matéria orgânica (\%) para três localidades da costa de Santa Catarina. Baía Sul (este trabalho), enseada da Armação do Itapocoroi (RESGALLA JR. et al., 2007) e enseada do Brito e de Bombinhas (SUPLICY et al., 2003).

Um ponto de destaque para a taxa de filtração refere-se à estabilização desta taxa a partir da concentração do séston de $10 \mathrm{mg} \cdot \mathrm{L}^{-1}$ o que refletiu também na estabilização da taxa de ingestão. Estes resultados indicam que a ingestão máxima ocorre acima deste valor de TPMw, e, devido a taxa de rejeição ser constante e por volta de $0,4 \mathrm{mg} \cdot \mathrm{h}^{-1} \cdot \mathrm{g}^{-1}$, valores de séston inferiores a $10 \mathrm{mg} \cdot \mathrm{L}^{-1}$ podem limitar uma energia disponível para $\mathrm{o}$ crescimento $\mathrm{e}$ reprodução do berbigão. Para Cerastoderma edule, um bivalvo infaunal, Iglesias et al. (1996) observou que a taxa de filtração foi máxima em uma concentração do séston de $5 \mathrm{mg} \cdot \mathrm{L}^{-1}$, entretanto, para esta espécie a rejeição cresce com a concentração do séston. A concentração de séston de $10 \mathrm{mg} \cdot \mathrm{L}^{-1}$ poderia ser um limitante ao desenvolvimento de parte da população localizada no interior do baixio devido ao efeito de sombra causado pelos organismos localizados na margem como destacado por Silva e Souza (2003), mas para a confirmação desta hipótese, seriam necessários estudos detalhados sobre a variação do séston na área de estudo.

\section{AGRADECIMENTOS}

Os autores agradecem a Paulo R. Pezzuto e Gilberto C. Manzoni pela coleta e fornecimento dos exemplares de berbigão e de água do Saco dos Limões, sem os quais não seria possível a realização deste trabalho. Ao DEINFRA/SC pelo apóio financeiro no programa de monitoramento da Via Expressa Sul.

\section{REFERÊNCIAS}

ARAÚJO, CM y. 2001. Biologia reprodutiva do berbigão Anomalocardia brasiliana na reserva extrativista marinha do Pirajubaé. Tese de Doutorado. Instituto de Biociências, Universidade de São Paulo. 204 p.

BACON, GS, BA MACDONALD, JE WARD. 1998. Physiological responses of infaunal (Mya arenaria) and epifaunal (Placopecten magellanicus) bivalves to variations in the concentration and quality of suspended particles I. Feeding activity and selection. J. Exp. Mar. Biol. Ecol., 219:105-125.

BAYNE, BL \& RC NEWELL. 1983. Physiological energetics of marine mollusks. In: Salevddin, ASM \& KM Wilbur (eds.). The mollusks. Physiology Parte 1. Academic Press. New York. 4:407-515.

BAYNE, BL, DA BROWN, K BURNSS, DR DIXON, A IVANOVICI, DR LIVINGSTONE, DM LOWE, MN MOORE, ARD STEBBING \& J WIDDOWS. 1985. The effects of stress and pollution on marine animals. Praegez Publishers, New York. 384p.

BAYNE, BL, AJS HAWKINS \& E NAVARRO. 1988. Feeding and digestion in suspension-feeding bivalve molluscs: the relevance of physiological compensations. Amer. Zool., 28:147-159.

BOEHS, G. 2000. Ecologia populacional, Reprodução e Contribuição em Biomassa de Anomalocardia brasiliana (GMELIN,1791) (Bivalvia:Veneridae) na Baía de Paranaguá, Paraná, Brasil. Tese de Doutorado. Universidade Federal do Paraná, Departamento de Zoologia. 201p.

GRIFFITHS, CL \& RJ GRIFFITHS. 1987. Bivalvia. In: PANDIAN, TJ \& FJ VERNBERG (eds.). Animal energetics. Bivalvia through Reptilian. Academic Press. New York. 2: 1-88.

GROTTA, M \& JE LUNETTA. 1980. Ciclo sexual de Anomalocardia brasiliana (Gmelin,1791) do litoral da Paraíba. Rev. Nordestina Biol., 3:5-55. 
GROTTA, M \& JE LUNETTA. 1982. Reproductive physiological variation of Anomalocardia brasiliana (Gmelin, 1791) (Mollusca: Bivalvia) in different latitudes. Rev. Nordestina Biol., 5:21-28.

HAWKINS, AJS, E NAVARRO \& JIP IGLESIAS. 1980. Comparative allometries of gut-passage time, gut content and metabolic faecal loss in Mytilus edulis and Cerastoderma edule. Mar. Biol., 105:197-204.

HAWKINS, AJS, RFM SMITH, BL BAYNE \& M HERAI. 1996. Novel observations underlying the fast growth of suspension-feeding shellfish in turbid environments: Mytilus edulis. Mar. Ecol. Progr. Series, 131:179-190.

IGLESIAS, JIP, MB URRUTIA, E NAVARRO, P ALVAREZ-JORNA, X LARRETXEA, S BOUGRIER \& M HERAL. 1996. Variability of feeding processes in the cockle Cerastoderma edule (L.) in response changes in séston concentration and composition. J. Exp. Mar. Biol. Ecol., 197:121-143.

IGLESIAS, JIP, MB URRUTIA, E NAVARRO \& I IBARROLA. 1998. Measuring feeding and absorption in suspension-feeding bivalves: an appraisal of the biodeposition method. J. Exp. Mar. Biol. Ecol., 219:71-86.

MACDONALD BA, GS BACON \& JE WARD. 1998. Physiological responses of infaunal (Mya arenaria) and epifaunal (Placopecten magellanicus) bivalves to variations in the concentration and quality of suspended particles II. Absorption efficiency and scope for growth. J. Exp. Mar. Biol. Ecol. 219:127-141.

MELLO, LGM. 1999. Taxas de biodeposição e clareamento do mexilhão Perna perna em ambiente de cultivo. Dissertação de Mestrado. UFSC. 33p.

MONTI D, L FRENKIEL \& M MOUEZA. 1991. Demography and growth of Anomalocardia brasiliana in a mangrove, in Guadeloupe (French west Indies). J. Moll. Stud. 57:249-257.

NARCHI, W. 1972. Comparative studies functional morphology of Anomalocardia brasiliana (Gmelin, 1791) and Tivela macroides (Born, 1778) (Bivalvia: Veneriae). Bull. Mar. Sci.,22(3): 643670.

NARCHI, W. 1976. Ciclo anual de gametogênese de Anomalocardia brasiliana. Bol. Zool. Univ. S. Paulo., 1:331-350.

NAVARRO E \& JIP IGLESIAS. 1993. Infaunal filter-feeding bivalves and the physiological response to short-term fluctuations in food availability and composition. In: DAME, RF (ed.). Bivalve filter feeders in estuarine and coastal ecosystem processes. NATO ASI Series, G 33. 25-56p.

NAVARRO, E, JIP IGLESIAS, A PEREZ CAMACHO, V LABARTA \& R BEIRAS. 1991. The physiological energetics of mussels (Mytilus galloprovincialis Lmk) from different cultivation rafts in the ria the Arosa (Galicia, N.W. Spain). Aquaculture. 94:197212.

PESO, MC. 1980. Bivalves comestíveis da Baía de Todos os Santos: estudo quantitativo com especial referência a Anomalocardia brasiliana (Gmelin,1791) (Molusca: Bivalvia). Tese de Doutorado. Universidade Federal do Paraná. 174p.

PEZZUTO, PR \& AM ECHTERNACHT. 1999. Avaliação de impactos da construção da Via Expressa SC-SUL sobre o berbigão Anomalocardia brasiliana (Mollusca: Bivalvia) na Reserva Extrativista Marinha do Pirajubaé, (Florianópolis, SC Brasil). Atlântica, 21: 105-119.

PRINS, TC \& AC SMAAL. 1989. Carbon and nitrogen budgets of the mussel Mytilus edulis (L.) and the cockle Cerastoderma edule (L.) in relation to food quality. In: ROS, JD (ed.). Topics in Marine Biology. Sc. Mar. 53(2-3):477-482.

PRINS, TC, AC SMAAL \& AJ POUWER. 1991. Selective ingestion of phytoplankton by the bivalves Mytilus edulis (L.) and Cerastoderma edule (L.). Hydrobiol. Bull. 25(1):93-100.

RESGALLA JR., C, ES BRASIL \& LC SALOMÃO. 2006. Physiological rates in different classes of sizes of Perna perna (Linnaeus, 1758) submmited to experimental laboratory conditions. Braz. J. Biol. 66:325 - 336.

RESGALLA JR., C, ES BRASIL, K LAITANO \& RW REIS FILHO. 2007. Physioecology of the mussel Perna perna (Mytilidae) in southern Brazil. Aquaculture, 270:464 - 474.

RIOS, EC. 1994. Seashells of Brazil. Editora FURG, Rio Grande. $492 p$.

SCHAEFFER-NOVELLI, Y. 1976. Alguns aspectos ecológicos da população de Anomalocardia brasiliana (Gmelin, 1791), na praia do Saco da Ribeira, Ubatuba, São Paulo. Tese de Doutorado. Instituto de Biociências da Universidade de São Paulo, 119p.

SCHAEFEER-NOVELLI, Y. 1980. Análise populacional de Anomalocardia brasiliana (Gemelin, 1791), na praia do Saco da Ribeira, Ubatuba, São Paulo. Bol. Inst. Oceanogr. Univ. S. Paulo, 29(2):351-355.

SCHMITT, JF. 2002. Efeito de diferentes condições ambientais em áreas de cultivo sobre alimentação e biodeposição do mexilhão Perna perna. Dissertação de Mestrado. UFSC. 84pp.

SILVA E SOUSA, D. 2003. Dinâmica Populacional do berbigão Anomalocardia brasiliana (Gmelin, 1791) (Mollusca: Bivalvia: Veneridae) na Reserva Extrativista Marinha do Pirajubaé (Florianópolis, SC) Trabalho de conclusão de curso: Oceanografia, UNIVALI. 36p.

SMAAL, AC, APMA VONCK \& M BAKKER. 1997. Seasonal variation in physiological energetics of Mitilus edulis and Cerastoderma edule of different size classes. J. Mar. Biol. Ass. U.K., 77:817-838.

SUPLICY, FM, JF SCHMITT, NA MOLTSCHANIWSKYJ \& JF FERREIRA. 2003. Modeling of filter-feeding behavior in the brown mussel, Perna perna (L.) exposed to natural variations of seston availability in Santa Catarina, Brazil. J. Shellfish Res., 22(1):125-134.

Recebido: 01/04/2008

Aceito: 04/03/2009 